\title{
Extrarenal Rhabdoid Tumor of the Liver
}

National Cancer Institute

\section{Source}

National Cancer Institute. Extrarenal Rhabdoid Tumor of the Liver. NCI Thesaurus. Code C96847.

A rare, aggressive primary malig nant tumor of the liver that occurs in childhood. It is characterized by the presence of a diffuse infiltrate of undifferentiated cells with rhabdoid features. 